\title{
A novel transgenic mouse model for immunological evaluation of carcinoembryonic antigen-based DNA minigene vaccines
}

\author{
He Zhou, ${ }^{1}$ Yunping Luo, ${ }^{1}$ Masato Mizutani, ${ }^{1}$ Noriko Mizutani, ${ }^{1}$ Jürgen C. Becker, ${ }^{2}$ \\ F. James Primus, ${ }^{3}$ Rong Xiang, ${ }^{1}$ and Ralph A. Reisfeld ${ }^{1}$
}

1Department of Immunology, The Scripps Research Institute, La Jolla, California, USA. '2Department of Dermatology, Julius Maximilians University, Wuerzburg, Germany. ${ }^{3}$ Vanderbilt University Medical Center, Nashville, Tennessee, USA

\begin{abstract}
A lack of relevant animal models has hampered preclinical screening and critical evaluation of the efficacy of human vaccines in vivo. Carcinoembryonic antigen-A2Kb (CEA-A2Kb) double transgenic mice provide a biologically relevant model for preclinical screening and critical evaluation of human CEA vaccine efficacy in vivo, particularly because such animals are peripherally tolerant of CEA. We established the utility of this model by demonstrating that an oral DNA minigene vaccine induces effective HLA-A2-restricted, CEA-specific antitumor CTL responses. This finding is supported by three lines of evidence: (a) an effective HLA-A2restricted, $\mathrm{CEA}_{691}$-specific CTL response; (b) specific in vitro killing of CEA-A2Kb transduced MC-38 colon carcinoma cells; and (c) protective immunity induced in vaccinated mice against challenges of these tumor cells. Importantly, peripheral $\mathrm{T}$ cell tolerance against CEA in CEA-A2 Kb double transgenic mice was broken by the $\mathrm{CEA}_{691}(\mathrm{IMIGVLVGV}$ ) minigene vaccine. In conclusion, CEA-A2Kb double transgenic mice were demonstrated to be good candidates for in vivo testing of human CEA-based vaccines. This result suggests a potential for these vaccines in future human vaccine development. The feasibility of using nonmutated selfantigens as targets for therapeutic vaccinations was indicated, provided that such antigens are presented in an immunogenic context; that is, as a DNA minigene in a bacterial carrier system.
\end{abstract}

\section{Introduction}

CD8 ${ }^{+}$CTLs have the ability to specifically recognize and kill tumor cells. They recognize antigens (Ag's) in the form of short peptides, generally 8 to 10 amino acids long, presented on the cell surface by MHC class I molecules. These peptides, usually referred to as CTL epitopes, are generated in the cytosol of cells after proteolytic processing of antigens by the proteasome (1). The primary aim of tumor vaccines is to induce antitumor $\mathrm{CD}^{+} \mathrm{CTL}$ responses that can eradicate tumors and prevent their relapse. DNA vaccines provide an attractive approach to this goal, particularly because vaccines against tumor self-antigens, consisting of either killed tumor cells or recombinant proteins, proved to be inefficient for delivery of antigen into the MHC class I Ag-processing pathway (2). Consequently, the induction of a more effective antigen-specific immune response by DNA vaccines requires new strategies for optimization of the vaccine design, including novel approaches for vaccine delivery and effective antigen processing. Such strategies include the use of an oral carrier system with an attenuated strain of Salmonella typhimurium (dam-; AroA $\left.A^{-}\right)$,which delivers the DNA to secondary lymphoid organs $(3,4)$.

Nonstandard abbreviations used: antigen (Ag); carcinoembryonic antigen peptide 1 (CAP1); carcinoembryonic antigen (CEA); effector/target (E/T); magnetic-activated cell sorting (MACS).

Conflict of interest: R.A. Reisfeld is a consultant for EMD-Lexigen Research Center, Billerica, Massachusetts, and received partial funding for this research from this company as indicated in the Acknowledgments.

Citation for this article: J. Clin. Invest. 113:1792-1798 (2004).

doi:10.1172/JCI200421107.
The use of minigene vaccines is an attractive approach because of the ease of synthesis and manipulation of these vaccines. Moreover, in contrast to whole-gene vaccines, minigene vaccines can induce immune responses against specific Ag epitopes while avoiding the interference of nonrelevant Ag epitopes. Consequently, such vaccines lend themselves to in-depth studies of immunological mechanisms far more readily than do DNA vaccines encoding entire genes. The minigene strategy was reported to induce effective antitumor responses by an HLA-A2-restricted Melan-A peptide analog epitope (5) or by murine MHC class I molecule $\mathrm{H}-2 \mathrm{D}^{\mathrm{b}} / \mathrm{K}^{\mathrm{b}}$-restricted melanoma Ag epitopes (4).

Carcinoembryonic antigen (CEA), an oncofetal protein with a relative molecular mass of 180,000 , is considered to be an effective target for a DNA vaccine because it is overexpressed by most colorectal, gastric, and pancreatic carcinomas (6), by $50 \%$ of breast cancers, and by $70 \%$ of non-small cell lung carcinomas; it is expressed to a far lesser extent on normal epithelium and also on some fetal tissues (7). This increased expression of CEA also promotes an increase in intercellular adhesions that may contribute to metastasis (8). Circulating CEA can also be detected in the blood of most patients with CEA-positive tumors and has been used to monitor responses to therapy and disease progression. Because of the overexpession of CEA on a large number of epithelial tumor cells, a variety of whole CEA gene- or CEA peptidebased vaccines have been the research focus of several laboratories $(9-12)$ and used in clinical trials $(13,14)$.

Moreover, short peptides corresponding to HLA-A2-restricted CTL epitopes derived from CEA have been identified and were 
shown to contribute to the cytotoxicity against tumor cells in vitro $(9,15,16)$. These observations prompted us to adopt a minigene approach to developing CEA-specific DNA vaccines designed for optimal antitumor activity and to avoid the induction of immune responses that are cross-reactive with normal cells.

To this end, two different HLA-A2-restricted epitopes were selected: carcinoembryonic antigen peptide 1-6D (CAP1-6D) (YLSGADLNL) and CEA ${ }_{691}$ (IMIGVLVGV). CAP1-6D was selected because it is an agonist peptide for CAP-1 (9) and was shown to be more antigenic than CAP-1 (17). $\mathrm{CEA}_{691}$ was chosen because it was reported to be presented by HLA-A2-expressing tumor cells (15).

\section{Results}

A CEA minigene encoded by expression vectors is expressed in mammalian cells. To achieve optimal vaccine efficacy, three expression vectors were constructed based on the backbone of $\mathrm{pCMV} / \mathrm{ER} / \mathrm{Myc}$ (Figure 1A). To check gene expression, 293T cells were transfected with either pHI-myc, pHI-CAP1-6D-myc, or pHI-691-myc using Lipofectamine 2000 (Invitrogen). Protein expression was assessed by Western blotting of cell lysates with monoclonal anti-myc $\mathrm{Ab}$ (Invitrogen), and single bands with the expected molecular weight of $15 \mathrm{kDa}$ or $16 \mathrm{kDa}$ were detected (Figure 1B). A lysate from cells transfected with pHI-CAP1-6D-myc revealed patterns identical to those of $\mathrm{pHI}-691-m y c$ (data not shown). The vaccine vectors $\mathrm{pHI}$, pHI-CAP1-6D, and pHI-691 were generated by introducing a stop codon immediately downstream from the peptide coding sequences, so that mature peptides did not contain the myc epitope. Structures were confirmed by DNA sequencing, and empty pCMV vectors were also included for control purposes.

CEA $A_{691}$-specific, HLA-A2-restricted immunity is induced by a DNA vaccine encoding the PHI-691 CEA minigene. To verify that a specific immune response was induced by the minigene vaccines, vaccination vectors were transfected into doubly attenuated S. typhimurium (dam; $\left.A r o A^{-}\right)$by electroporation and used to vaccinate C57-CEA-A2Kb double transgenic mice three times at 2 -week intervals. To this end, ELISPOT assays were performed 2 weeks after the last immunization with splenocytes isolated from immunized mice; synthetic peptides $(10 \mu \mathrm{g} / \mathrm{ml})$ were applied as stimulators. All experimental groups revealed an increased number of spots when splenocytes were stimulated with the $\mathrm{CEA}_{691}$ peptide, compared with unstimulated splenocytes. Although this increase is minimal in the groups of mice immunized with either the PCMV, pHI, or PHI-CAP1-6D DNA vaccines, it is quite dramatic in the mice vaccinated with PHI-691 (Figure $2 \mathrm{~A}$ ), which suggests the existence of $\mathrm{CEA}_{691}$-specific cells that can be induced to secrete IFN- $\gamma$ in C57-CEA-A2Kb double transgenic mice.

However, in all experimental groups, stimulation of splenocytes with the CAP1-6D peptide failed to significantly increase the number of spots compared with unstimulated splenocytes; that is, CAP1-6D-specific, IFN- $\gamma$-secreting cells could not be detected. This finding suggests that CAP1 may not be a dominant Ag epitope in CEA-A2 Kb double transgenic C57BL/6J mice, despite the fact that it is one of the more dominant HLA-A2-restricted, CEA-specific epitopes in humans $(9,17)$. This notion is further supported by the finding that no CAP1-6D-specific, IFN- $\gamma$-secreting cells were detected in mice immunized with a vector encoding the full-length CEA gene (data not shown).

To confirm that the $\mathrm{CEA}_{691}$-specific immune response induced was indeed HLA-A2 restricted, splenocytes from vaccinated mice were also cultured in vitro in the presence of irradiated (1,000 Gy) MC-38-CEA-A2 Kb cells for 5 days and thereafter used in ELISPOT assays. HLA-A2 ${ }^{+}$human T2 cells deficient in transporter associated with antigen processing (TAP) were used as stimulators. Such cells were used either unloaded or loaded overnight with $10 \mu \mathrm{g} / \mathrm{ml}$ CAP1-6D or $\mathrm{CEA}_{691}$ peptides and irradiated (1,000 Gy). In mice immunized with the pHI-691 DNA minigene vaccine, an increased number of IFN- $\gamma$-secreting cells was detected when splenocytes from such mice were stimulated with $\mathrm{CEA}_{691}$-loaded $\mathrm{T} 2$ cells. This increase was particularly evident when compared with splenocytes that were stimulated with either unloaded or CAP1-loaded T2 cells (Figure 2B). Similar results were obtained by substituting T2 cells with another HLA-A2+ cell line, B3 (data not shown). B3 is an EBV-transformed human B cell line established in our laboratory from a normal individual, which maintained high levels of HLA-A2 expression (Figure 2C). Because HLA-A2 is the only MHC molecule in common among the stimulating T2 or B3 cells of human origin and the responding CEA-A2Kb transgenic mouse splenocytes, these data suggest that the $\mathrm{CEA}_{691}$-specific reaction observed is indeed HLA-A2 restricted.

When compared with those with no stimulation added to the splenocytes, the addition of irradiated $\mathrm{T} 2$ cells by themselves induced more splenocytes to secrete IFN- $\gamma$ in every experimental group tested. However, this finding is not surprising because of the human origin of 2 cells. This reaction was stronger in the $\mathrm{pHI}$ group than in the pCMV vaccine group of mice, which suggests that $\mathrm{pHI}$ is an effective immune stimulator. Moreover, stimulation with $\mathrm{CEA}_{691}$-loaded T2 cells failed to induce more cells to secrete IFN- $\gamma$ in the pCMV, pHI, and $\mathrm{PHI}-\mathrm{CAP} 1-6 \mathrm{D}$ experimental groups of mice. This failure may be

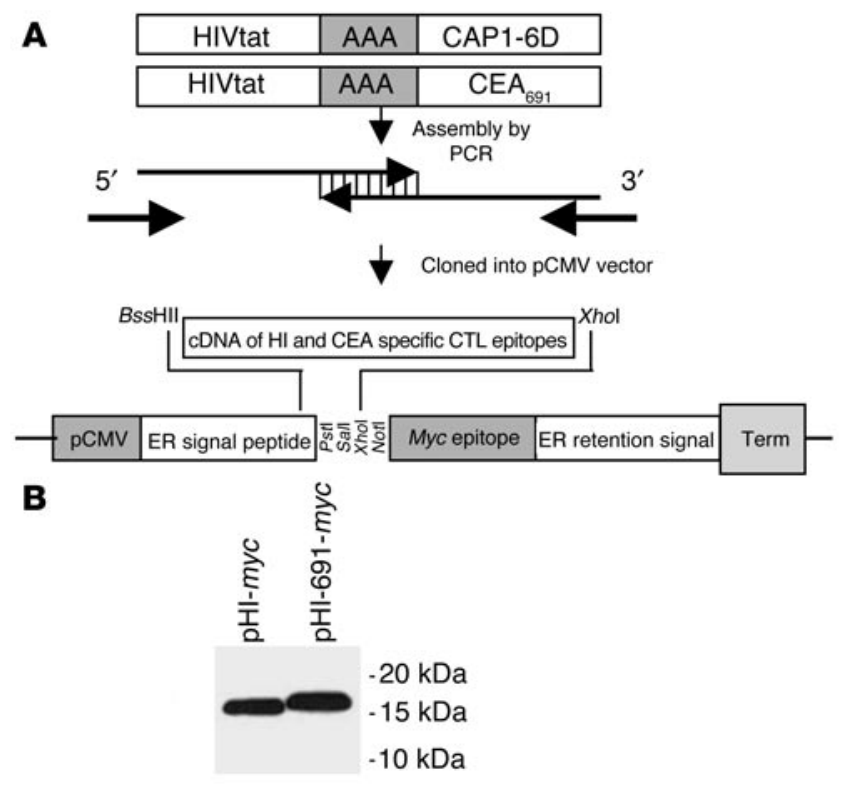

\section{Figure 1}

Expression vectors are constructed and verified. (A) Schematic map of vector constructs. Minigenes encoding HIVtat translocation peptide, a spacer, and human CEA epitopes CAP1-6D or CEA 691 were assembled by PCR with overlapping oligonucleotides as templates. The PCR fragments generated were cloned into a pCMV vector by using Bss $\mathrm{HII}$ and $\mathrm{Xhol}$ restriction sites. (B) Proteins encoded by minigenes were expressed in mammalian cells. This expression was indicated when 293T cells were transfected with either $\mathrm{pHI}-m y c$ or $\mathrm{pHI}-691-m y c$ for 24 hours, harvested, lysed, and analyzed by Western blotting with monoclonal antibody against myc. 
A

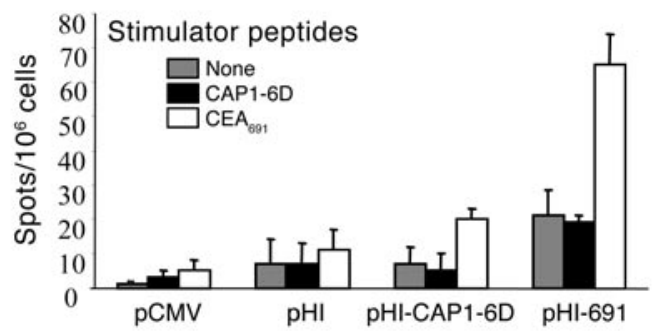

B

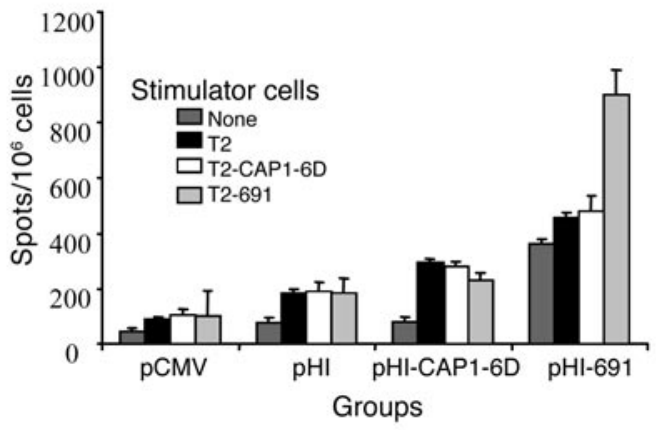

C

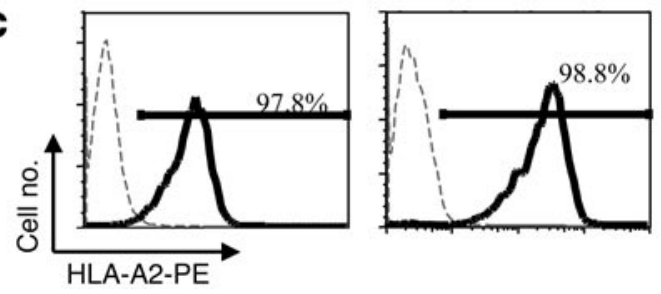

caused by the dominance of the responses induced against $\mathrm{T} 2$ cells, which are xenogeneic, except for their HLA-A2 expression.

HLA-A2-restricted, $C E A_{691}-$ specific responder cells are cytotoxic. The fact that the HLA-A2-restricted, $\mathrm{CEA}_{691}$-specific responder cells are cytotoxic was confirmed with standard ${ }^{51} \mathrm{Cr}$-release assays performed with splenocytes isolated from immunized mice. Target cells included CAP1-6D-loaded or $\mathrm{CEA}_{691}$-loaded T2 cells. CAP1-6Dloaded T2 cells were used as a control because CAP1-6D-specific cells could not be detected in ELISPOT assays. Splenocytes obtained from the pHI-691 experimental group of mice exhibited significantly higher killing of $\mathrm{CEA}_{691}$-loaded $\mathrm{T} 2$ cells than did splenocytes from $\mathrm{pCMV}, \mathrm{pHI}$, and $\mathrm{pHI}-\mathrm{CAP} 1-6 \mathrm{D}$ control groups $(P<0.0005, P<0.0005$, and $P<0.005$ at an effector/target $[\mathrm{E} / \mathrm{T}]$ ratio of 100:1, respectively [Figure 3A]). However, when T2 cells loaded with CAP1-6D were used as targets, the cytotoxic activity of splenocytes isolated from the pHI-691-immunized group was less significant compared with that of mice vaccinated with either $\mathrm{pHI}$ or pHI-CAP1-6D $(P<0.05$ and $P>0.05$ at an $\mathrm{E} / \mathrm{T}$ ratio of $100: 1$, respectively [Figure $3 \mathrm{~B}]$ ), which indicates the specificity of this cytotoxic activity. As previously mentioned, because both effector

\section{Figure 3}

HLA-A2-restricted, CEA 691 -specific T cells induced by the pHI-691 minigene vaccine are cytotoxic. Groups of C57BL/6-CEA-A2Kb mice $(n=4)$ were immunized three times at 2-week intervals with attenuated $S$. typhimurium harboring the vectors indicated. Mice were sacrificed 2 weeks after the last immunization, and isolated splenocytes were stimulated with irradiated MC-38-CEA-A2Kb cells for 5 days. Cytotoxicity assays were performed with (A) $\mathrm{CEA}_{691}$-loaded T2 cells or (B) CAP1-6D-loaded T2 cells as targets.

\section{Figure 2}

An HLA-A2-restricted, $\mathrm{CEA}_{691}$-specific response is induced by the pHI-691 DNA minigene vaccine. Groups of C57BL/6-CEA-A2Kb mice $(n=4)$ were immunized three times at 2-week intervals with attenuated S. typhimurium harboring the vectors indicated. Two weeks after the last immunization, mice were sacrificed and ELISPOT assays performed on splenocytes isolated by using synthetic peptides $(10 \mu \mathrm{g} / \mathrm{ml})$ as stimulators (A). The remaining splenocytes were stimulated with irradiated MC-38-CEA-A2Kb cells for 5 days, and ELISPOT assays were then performed using either irradiated unloaded or peptide-loaded T2 cells as stimulators (B). (C) HLA-A2 expression of T2 (left) and $B 3$ (right) cells. B3 is an EBV-transformed cell line generated from a healthy HLA-A2+ individual as described in Methods. Cells were treated with control antibody (thin dashed lines) or anti-HLA-A2 antibody (thick solid lines) and then stained with PE-conjugated goat anti-mouse Ig.

mouse splenocytes and human T2 target cells only share HLA-A2, this specific cytotoxicity might be HLA-A2 restricted as well.

$C D 8^{+} T$ cells mediate HLA-A2-restricted cytotoxic killing. To verify that the induced HLA-A2-restricted, $\mathrm{CEA}_{691}$-specific killing was mediated by $\mathrm{CD}^{+} \mathrm{T}$ cells, freshly isolated splenocytes were fractionated into $\mathrm{CD}^{+}$and $\mathrm{CD}^{-}$subpopulations by magnetic-activated cell sorting (MACS). The purity of the cell fractions is shown in Figure 4A. These fractionated cells were stimulated with irradiated MC-38-CEA-A2 Kb in the presence of IL-2 for 5 days, and then standard ${ }^{51} \mathrm{Cr}$-release assays were performed using $\mathrm{CEA}_{691}$-loaded T2 cells as targets. Killing of target cells was only observed with the $\mathrm{CD}^{+}$population, whereas the $\mathrm{CD} 8^{-}$population showed hardly any killing at all (Figure $4 \mathrm{~B}$ ). The $\mathrm{CD} 8^{+}$population from the pHI-691 experimental group of mice showed higher cytotoxicity than that of the pHI-CAP1-6D group, which was used as a control group in this experiment $(P<0.005, P<0.005$, and $P<0.05$ at $\mathrm{E} / \mathrm{T}$ ratios of 20:1, 10:1, and 5:1, respectively [Figure $4 C$ ]).

When $\mathrm{CD}^{+}$cells were enriched by MACS, the percentage of $\mathrm{CD}^{+} \mathrm{T}$ cells increased from approximately $30 \%$ in the unfractionated culture to more then $90 \%$ in the $\mathrm{CD}^{+}$cell culture. However, the $\mathrm{CD}^{+}$fraction did not show higher killing rates (Figure $4 \mathrm{C}$ ) when compared with unfractionated splenocytes (Figure 3A), presumably because of the requirement of CD8- $\mathrm{T}$ cells for optimal proliferation in cell culture. One line of evidence supporting this notion is that culture of the $\mathrm{CD}^{+}$fraction resulted in lower CD8 expression when compared with the unfractionated cultured splenocytes (data not shown).
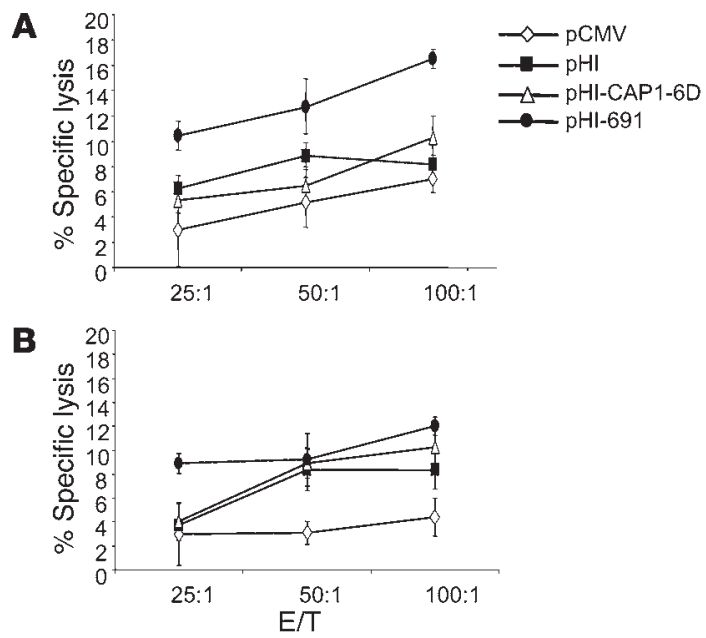
A

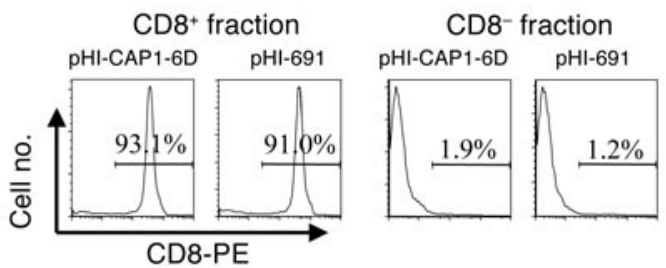

B

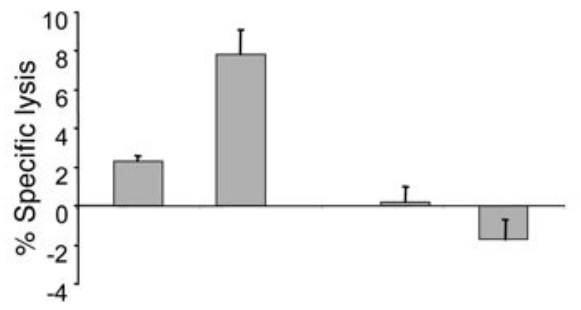

C

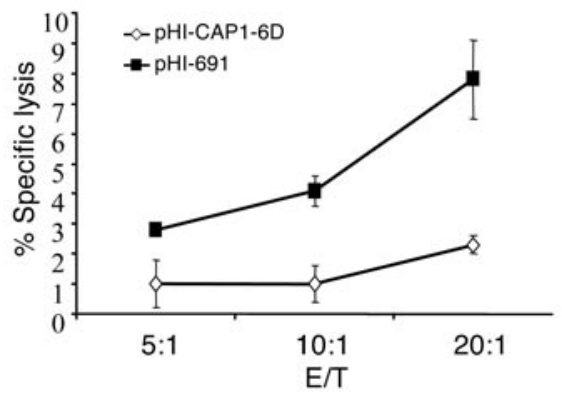

Taken together, these data indicate that the $\mathrm{pHI}-\mathrm{CEA}_{691}$ minigene vaccine can effectively induce an HLA-A2-restricted, CEA ${ }_{691-}$ specific CTL response in CEA-A2Kb double transgenic mice.

Protective immunity against CEA-A2Kb-transduced colon carcinoma cells. HLA-A2-restricted, $\mathrm{CEA}_{691}$-specific CTLs, induced by the pHI-691 minigene DNA vaccine, recognized and killed CEA-A2Kb doubly transduced MC-38 murine colon carcinoma cells, that is, MC-38CEA-A2Kb cells, whose surface expression of HLA-A2 and CEA is depicted in Figure 5A (left). This process was demonstrated by isolating splenocytes from mice 2 weeks after the last immunization and stimulating them in vitro with irradiated MC-38-CEA-A2Kb cells for 5 days and then testing them in a standard ${ }^{51} \mathrm{Cr}$-release assays. As expected, splenocytes from the group of mice immunized with the pHI-691 vaccine showed the most effective cytotoxic killing of MC-38-CEA-A2 Kb target cells when compared with PCMV, $\mathrm{pHI}$, and pHI-CAP1-6D control groups $(P<0.0005, P<0.001$, and $P<0.005$ at an E/T ratio of 100:1, respectively [Figure 5B]). This enhanced killing ability appeared to be HLA-A2 restricted because the killing percentage of the HLA-A2- MC-38-CEA cells (HLA-A2

\section{Figure 5}

The DNA minigene vaccine $\mathrm{pHI}-691$ induces HLA-A2-restricted killing of MC-38-CEA-A2Kb cells. (A) Surface expression of HLA-A2 and CEA by MC-38-CEA-A2Kb (left) and MC-38-CEA (right). Tumor cells were washed and incubated with isotype control $A b$ (thin dashed lines), anti-HLA-A2 (thin solid lines), or anti-CEA (heavy solid lines) and stained with PE-conjugated $\left(\mathrm{Fab}^{\prime}\right)_{2}$ of goat anti-mouse Ig Ab. Groups of C57BL/6-CEA-A2Kb mice $(n=4)$ were immunized three times at 2-week intervals with attenuated $S$. typhimurium harboring the vectors indicated. Mice were sacrificed 2 weeks after the last immunization, and isolated splenocytes were stimulated with irradiated MC-38-CEA-A2Kb cells for 5 days. Thereafter, cytotoxicity assays were performed with (B) MC-38-CEA-A2Kb or (C) MC-38-CEA as target cells. max, maximum.

\section{Figure 4}

DNA minigene-induced cytotoxicity is mediated by $\mathrm{CD} 8^{+} \mathrm{T}$ cells. Groups of C57BL/6-CEA-A2Kb mice $(n=4)$ were immunized three times at 2-week intervals with attenuated $S$. typhimurium harboring either pHI-CAP1-6D or pHI-691 vectors. Mice were sacrificed 2 weeks after the last immunization, and isolated splenocytes were fractionated into $\mathrm{CD}^{+}$or $\mathrm{CD}^{-}$populations. Each cell population was stimulated with irradiated MC-38-CEA-A2Kb cells for 5 days. Thereafter, cytotoxicity assays were performed with $\mathrm{CEA}_{691}$-loaded $\mathrm{T} 2$ cells as targets. (A) Purity of each subpopulation after MACS. (B) Cytotoxicity of each cell population at E/T ratio of 20:1. (C) Cytotoxicity of CD8 ${ }^{+}$ populations is shown at different $E / T$ ratios.

and CEA expression shown in Figure 5A [right]) or parental MC38 target cells did not differ significantly among the experimental groups $(P>0.05$ at an $\mathrm{E} / \mathrm{T}$ ratio of 100:1 [Figure 5C and data not shown]). Taken together, these data suggest that those immune cells that presumably are HLA-A2 restricted and $\mathrm{CEA}_{691}$-specific CTLs, induced by the $\mathrm{pHI}-691$ vaccine, are indeed capable of recognizing and killing CEA-A2 $\mathrm{Kb}$ doubly transfected MC-38 cells in vitro.

To test the specificity of this immune response, splenocytes isolated from $\mathrm{pHI}-691$ vaccinated group of mice were stimulated weekly with irradiated MC-38-CEA-A2Kb cells. These cells, after being restimulated four times, were more than $86 \% \mathrm{CD}^{+}$as demonstrated by flow cytometry (Figure 6A). These stimulated cells were then harvested and analyzed by ${ }^{51} \mathrm{Cr}$-release assays against either unloaded or peptide-loaded HLA-A ${ }^{+} \mathrm{B} 3$ cells. In ${ }^{51} \mathrm{Cr}$-release assays, the cytotoxic killing efficacy of $\mathrm{CEA}_{691}$-loaded $\mathrm{B} 3$ cells was greatly enhanced; that is, a much higher killing percentage was observed at lower $\mathrm{E} / \mathrm{T}$ ratios, with the specificity still being maintained as indicated by the lower percentage of specific killing of unloaded B3 cells (Figure 6B).

The presence of $\mathrm{T}$ cells able to kill tumor cells in the circulation of vaccinated animals suggests that this $\mathrm{T}$ cell response may also be effective in vivo, that is, protecting mice from a tumor challenge. To test this hypothesis, mice were challenged subcutaneously in the right flank with $5 \times 10^{5} \mathrm{MC}-38$-CEA-A2Kb cells 2 weeks after the last immunization. These animals were sacrificed 28 days later, and tumor weights were assessed. In all groups of experimental animals, tumor size varied considerably within each group. This
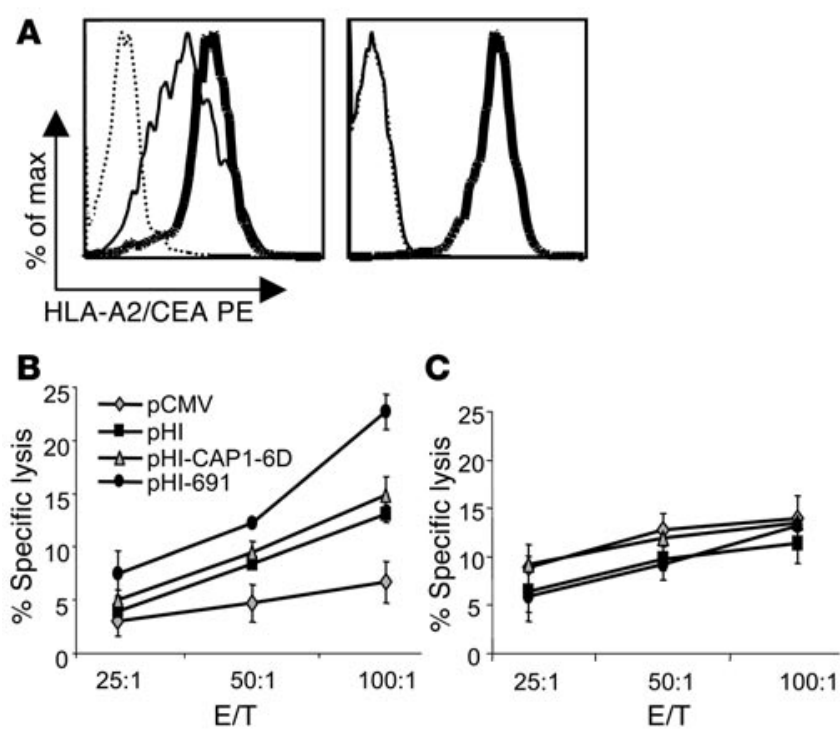

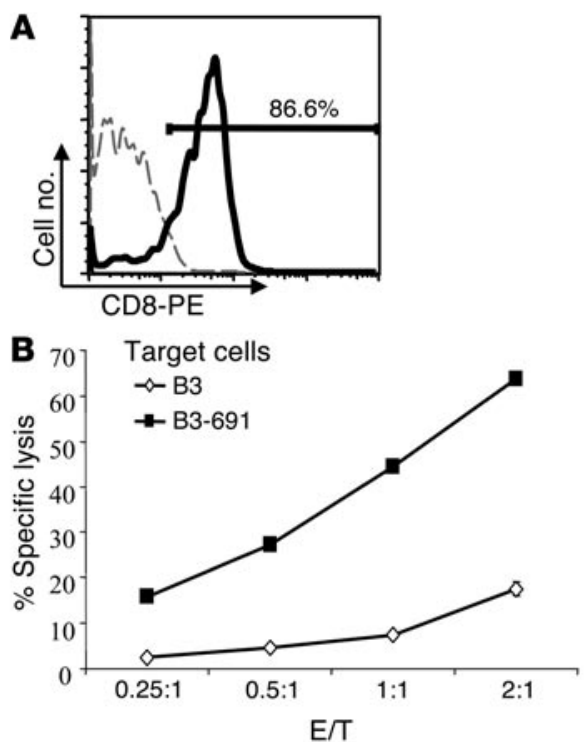

finding is not too surprising because of the complex genetic background of the doubly transgenic mice and tumor cell lines. However, the average tumor size was still reduced in the pHI-691 group, and the difference reached significance when compared with the pCMV group $(P<0.02$ [Figure 7A]). Importantly, 3 of 10 mice rejected the tumor challenge completely, whereas all mice in the pCMV, pHI, and pHI-CAP1-6D experimental groups uniformly developed tumors (Figure 7A). This experiment was repeated with similar results (data not shown). The protection by the minigene vaccine is specific for MC-38-CEA-A2Kb tumor cells as well as HLA-A2 restricted because no protection was observed against parental MC-38-CEA and MC-38 tumor cells (Figure 7B).

\section{Discussion}

The availability of CEA transgenic mice expressing CEA as a self-antigen with a tissue distribution similar to that of humans (18-20) provides a particularly relevant model for critically evaluating the potential efficacy of a human CEA-based vaccine because these animals developed peripheral tolerance to CEA. This finding is particularly evident from the lack of detectable, CEA-specific serum antibodies and the inability to prime an in vitro splenic $T$ cell response to CEA. These transgenic mice already proved valuable for thorough evaluation of DNA vaccines encoding the entire CEA gene or for CEApeptide vaccines per se $(10,21,22)$. However, because human MHC class I alleles are distinct from those of mice, animals transgenic for only CEA were inadequate for testing HLA-A2-restricted minigene

\section{Figure 7}

DNA minigene vaccine $\mathrm{pH}-691$ exhibits HLA-A2-restricted antitumor effects in CEA-A2Kb double transgenic mice. Groups of mice $(n=$ 7-10) were immunized three times at 2-week intervals with attenuated S. typhimurium harboring the vectors indicated. Mice were challenged 2 weeks after the last immunization subcutaneously with $5 \times 10^{5}$ MC-38-CEA-A2Kb, MC-38, or MC-38-CEA cells. Mice were sacrificed 28 days later and tumor weights assessed. (A) MC-38-CEA-A2Kb tumor weight, the horizontal bar showed the average. (B) Average tumor weight of each group of mice $(n=8)$ challenged with different tumor cells. Top shows MC-38 tumor size; bottom shows average MC-38CEA tumor weight. ${ }^{*} P<0.02$ compared with $\mathrm{PCMV}$ group.

\section{Figure 6}

Repetitive stimulation with MC-38-CEA-A2Kb cells results in enriched HLA-A2-restricted, CEA 691 -specific CTLs. C57BL/6-CEA-A2Kb mice $(n=4)$ were immunized three times at 2-week intervals with attenuated S. typhimurium harboring the $\mathrm{pHI}-691$ vector. Mice were sacrificed 2 weeks after the last immunization, and isolated splenocytes were stimulated weekly with irradiated MC-38-CEA-A2Kb cells. After four stimulations, cells were harvested and cytotoxicity assays performed. (A) Expression of CD8 on stimulated cells. Stimulated splenocytes were stained with $\mathrm{PE}$-conjugated isotype control antibody (thin dashed line) or anti-CD8 Ab (thick solid line). (B) Cytotoxicity assays performed with either unloaded or $\mathrm{CEA}_{691}$-loaded $\mathrm{B} 3$ cells as targets.

vaccines, and HLA-A2Kb transgenic mice were required. Such mice express $\alpha_{1}$ and $\alpha_{2}$ domains of the human HLA-A2.1 molecule, as well as the $\alpha_{3}$ domain from the mouse $\mathrm{H}-2 \mathrm{~K}^{\mathrm{b}}$ molecule (23). Indeed, mice transgenic for HLA-A2 were shown to be useful for studying human antigens per se and the immune response directed against them (12, 23-25). However, mice transgenic only for HLA-A2 are unsuitable for in vivo studies of HLA-A2-restricted, CEA-specific responses because such animals still recognize CEA as a foreign antigen and lack peripheral $\mathrm{T}$ cell tolerance against CEA. Consequently, for the studies reported here, CEA-A2Kb double transgenic mice were generated by breeding transgenic mice homozygous for CEA and HLA$\mathrm{A} 2 \mathrm{~Kb}$. Because such animals express human CEA antigen presented by human HLA-A2 molecules, they represent a most relevant in vivo animal model to study human immune responses to DNA-based CEA minigene vaccines and can be used for in vivo preclinical evaluations of the vaccines for specificity and therapeutic efficacy. Importantly, as predicted, the $\mathrm{CEA}-\mathrm{A} 2 \mathrm{~Kb}$-transduced colon carcinoma cell line MC-38 is tumorigenic in these CEA-A2Kb double transgenic mice; thus, it was possible to test the effectiveness of the applied vaccination in vivo. To our knowledge, our study is the first to utilize $\mathrm{CEA}-\mathrm{A} 2 \mathrm{~Kb}$ double transgenic mice for such in vivo studies.
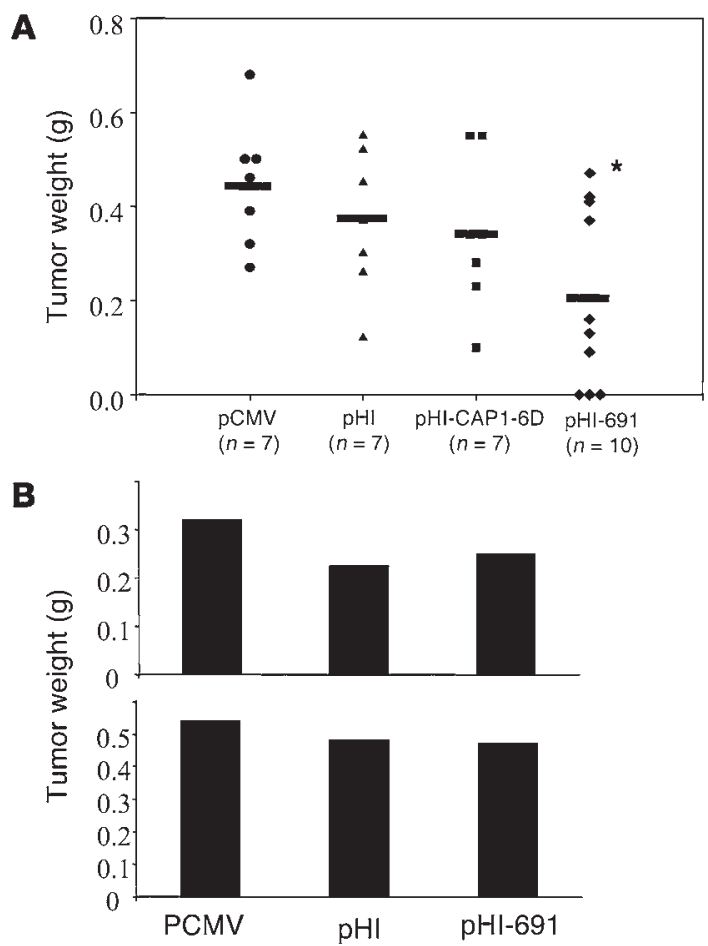
We tested two different HLA-A2-restricted CEA epitopes with our DNA minigene vaccines: CAP1-6D (YLSGADLNL) $(9,17)$ and CEA $_{691}$ (IMIGVLVGV) (15). The DNA minigene vaccine pHI-691 clearly induced a relevant HLA-A2-restricted, CEA 691 -specific CTL response similar to the response to this same CEA-peptide reported in humans (15). The biological relevance of this animal model was confirmed, because in CEA-A2Kb double transgenic mice, these CTLs specifically killed CEA-A2Kb-transduced MC-38 cells in vitro and protected mice from a challenge of these tumor cells in vivo.

It should be noted that the CEA-A2 Kb transgenic mice still harbor a murine $\mathrm{T}$ cell receptor repertoire. In this regard, Theobald et al. (26) have demonstrated that a correlation between an immune response to some Ag epitopes observed in HLA-A2 transgenic mice and in vitro immune responses in humans does not necessarily exist. Consequently, whether this phenomenon is responsible for the failure of the $\mathrm{PHI}-\mathrm{CAP} 1-6 \mathrm{D}$ DNA minigene vaccine to induce a CAP1-6D-specific CTL response or whether the pHI-CAP1-6D DNA minigene vaccine itself is insufficient to induce such response deserves further investigation.

The rationale for using the HIVtat peptide, which is derived from the HIVtat Ag, in our minigene vaccine is based on the fact that it is one of the commonly used membrane-translocating peptides. These peptides are able to transport other peptides into the endoplasmic reticulum (ER) in a TAP-independent manner, where they can be processed and trimmed to CTL epitopes (27-29). Such peptides can be internalized in a receptor-independent and energy-independent fashion; however the exact mechanism by which they can translocate across the plasma membrane into the cytoplasm remains unknown (29). In our vaccine, $\mathrm{CEA}_{691}$ was cloned downstream from the HIVtat peptide to ensure its proper delivery and processing in the proteasome. In this regard, Lu et al. (29) demonstrated that by linking to the HIVtat peptide, Ag epitope peptide can be transported into the cell to form complexes with MHC class I molecules in the ER and the trans-Golgi region. In addition, an orally delivered DNA minigene vaccine against murine melanoma (i.e., a plasmid encoding only peptides) was shown to be ineffective in inducing an epitope-specific response (4). When attenuated S. typhimurium, harboring vector encoding solely the $\mathrm{CEA}_{691}$ peptide, were used to immunize mice, they induced a $\mathrm{CEA}_{691}$-specific response detected by ELISPOT assays; however, this immune response was not as effective as that obtained with the pHI-691 vaccine (data not shown).

In summary, our data demonstrate that peripheral $\mathrm{T}$ cell tolerance against the CEA self-antigen could be broken by our pHI691 DNA minigene vaccine in CEA-A2 Kb double transgenic mice. This ability is indicated by an effective HLA-A2-restricted, CEA $61^{-}$ specific CTL response, which can specifically kill CEA-A2Kb transduced MC- 38 colon carcinoma cells in vitro and protect mice from a challenge by these tumor cells in vivo. This DNA minigene vaccine, thus, provides an interesting alternative design of CEAbased DNA vaccines for potential clinical use.

\section{Methods}

Animals, bacterial stains, and cell lines. C57BL/6J-CEA and C57BL/6J$\mathrm{A} 2 \mathrm{~Kb}$ homozygous transgenic breeder mice were generated as previously described $(18,30)$. C57BL/6J-CEA-A2Kb double transgenic mice were generated in the animal facility of The Scripps Research Institute by crossing male C57BL/6J-CEA with female C57BL/6J-A2Kb mice. The genotype of such mice was confirmed by PCR and flow cytometry, respectively. All animal experiments were performed according to the NIH Guides for the Care and Use of Laboratory Animals.
The doubly attenuated S. typhimurium AroA- and dam ${ }^{-}$strain RE88 was kindly provided by Remedyne Corporation (Santa Barbara, California, USA). The murine colon carcinoma cell line MC-38-CEA was obtained as described before (18). MC-38-CEA-A2Kb cells were generated by transfecting MC-38-CEA cells with a plasmid encoding A2Kb. All cell lines were cultured in DMEM (Gibco; Invitrogen Corp., Grand Island, New York, USA) supplemented with 10\% (vol/vol) FBS. MC-38-CEA cells were cultured in the presence of $1 \mathrm{mg} / \mathrm{ml} \mathrm{G} 418$ (Sigma-Aldrich, St. Louis, Missouri, USA), and MC-38-CEA-A2Kb cells were cultured with G418 and $0.5 \mathrm{mg} / \mathrm{ml}$ Zeocin (Invitrogen, Carlsbad, California, USA). T2, a human HLA-A2 ${ }^{+}$cell line, was originally obtained from P. Cresswell (Yale University, New Haven, Connecticut, USA) and kindly provided by L.A. Sherman (The Scripps Research Institute). Another HLA-A2+ cell line, B3, was established by transforming PBMCs from a healthy HLA-A2 ${ }^{+}$individual with EBV.

Construction of expression vectors. The expression vector $\mathrm{PCMV/}$ $\mathrm{ER} / \mathrm{Myc}$ was purchased from Invitrogen. Vector construction is illustrated schematically in Figure 1A. The following expression vectors were constructed: pHI-myc, pHI-CAP1-6D-myc, and pHI691-myc, where HIVtat peptide represents RKKRRQRRR, CAP16D peptide represents YLSGADLNL (17), and CEA $_{691}$ peptide represents IMIGVLVGV (15). All peptides were engineered to be in-frame with the myc epitope. Constructs were confirmed by DNA sequencing at The Scripps Research Institute's Core Facility (La Jolla, California, USA). Peptide expression was demonstrated by Western blotting with monoclonal anti-myc antibody (Invitrogen). Once peptide expression was verified, a stop codon was introduced immediately in front of the myc epitope sequences. The resulting vectors, namely pHI, pHI-CAP1-6D, and pHI-691, were verified by sequencing and used to transform doubly attenuated S. typhimurium $\left(\mathrm{dam}^{-} ;\right.$Aro $\left.A^{-}\right)$for immunization. The $\mathrm{pCMV}$ empty vector was also included in the experiments as a control.

Transformation of S. typhimurium. Doubly attenuated S. typhimurium $\left(\right.$ dam $^{-} ;$Aro $\left.^{-}\right)$were transformed with DNA vaccine plasmids by electroporation. Briefly, freshly prepared bacteria $\left(1 \times 10^{8}\right)$, at mid$\log$ growth phase, were mixed with plasmid DNA ( 1 to $2 \mu \mathrm{g}$ ) on ice in a $0.1-\mathrm{cm}$ cuvette and electroporated at $2.0 \mathrm{kV}, 25 \mu \mathrm{F}$, and $100 \Omega$.

Peptide synthesis. The CAP1-6D peptide, with greater than $95 \%$ purity by HPLC, was a gift from J. Schlom (National Cancer Institute, Bethesda, Maryland, USA). The $\mathrm{CEA}_{691}$ peptide was synthesized by Multiple Peptide System (San Diego, California, USA) with greater than $93 \%$ purity by HPLC.

Oral immunization and tumor cell challenge. Groups of C57BL/6JCEA-A2 Kb mice $(n=7-10)$ were immunized three times at 2-week intervals by gavage with $100 \mu \mathrm{l}$ PBS that contained approximately $5 \times 10^{8}$ doubly mutated $S$. typhimurium harboring either pCMV, pHI, pHI-CAP1-6D, or pHI-691. Mice were challenged subcutaneously in the right flank with $5 \times 10^{5} \mathrm{MC}-38$-CEA-A2Kb colon carcinoma cells 2 weeks after the last immunization. The subcutaneous tumors were monitored daily. Mice were sacrificed 28 days after tumor challenge, and tumor weights were determined and compared with those of control subjects.

Cytotoxicity assay. Cytotoxicity was measured by a standard chromium-51 $\left({ }^{51} \mathrm{Cr}\right)$ release assay as previously described, with some modification (31). Briefly, splenocytes were harvested 2 weeks after the last immunization and stimulated in vitro by irradiated $(1,000$ Gy) MC-38-CEA-A2Kb cells at $37^{\circ} \mathrm{C}$ for 5 days in RPMI 1640 supplemented with $10 \%$ FBS, L-glutamine, 15 mM HEPES, nonessential amino acids, sodium pyruvate, 2-ME, and recombinant IL-2 at $20 \mathrm{U} / \mathrm{ml}$ (PeproTech, Rocky Hill, New Jersey, USA). Splenocytes 
were harvested and separated with Lympholyte-M cell separation media (Cedarlane Laboratories Ltd., Hornby, Ontario, Canada). Target cells were labeled with ${ }^{51} \mathrm{Cr}$ for 1.5 hours at room temperature and incubated with effector cells at various effector-to-target cell ratios at $37^{\circ} \mathrm{C}$ for 4 hours. The percentage of specific target cell lysis was calculated with the formula $[(E-S) /(T-S)] \times 100$, where $E$ is the average experimental release, $S$ is the average spontaneous release, and $T$ is the average total release.

ELISPOT assays. ELISPOT assays were performed with an ELISPOT kit (BD Biosciences Pharmingen, La Jolla, California, USA) according to the instructions provided by the manufacturer. Briefly, splenocytes were isolated 2 weeks after the last immunization. After lysis of the red blood cells with ACK lysis buffer (Cambrex Bio Science, Walkersville, Maryland, USA), splenocytes were resuspended at a final concentration of $2 \times 10^{7}$, and $100 \mu \mathrm{l}$ of this suspension was then incubated with $100 \mu \mathrm{l}$ medium with or without the peptides or irradiated stimulator cells overnight at $37^{\circ} \mathrm{C}$ in ELISPOT plates coated with anti-IFN- $\gamma$. In some cases, splenocytes were stimulated with irradiated (1,000 Gy) MC-38-CEA-A2Kb cells for 5 days, and ELISPOT assays were performed thereafter.

$M A C S$ and flow cytometry. MACS was performed with anti-mouse $\mathrm{CD} 8 \mathrm{Ab}$ conjugated magnetic beads (Miltenyi Biotec, Sunnyvale, California, USA) according to the manufacturer's instructions. The purity of fractionated cell populations was checked by flow cytometry using phycoerythrin-conjugated (PE-conjugated) antimouse CD8 Ab. Flow cytometry was performed with $1 \times 10^{6}$ cells on a FACSCalibur (Becton Dickinson, San Jose, California, USA) equipped with a CellQuest program. Analyses were performed with FlowJo software (Tree Star Inc., Ashland, Oregon, USA). Other antibodies used in such analyses included purified anti-HLA-A2 $\mathrm{Ab}$ (BD Biosciences Pharmingen), purified anti-CEA Ab (ICN Biomedicals Inc., Aurora, Ohio, USA), and PE-conjugated $\mathrm{F}\left(\mathrm{ab}^{\prime}\right)_{2}$ of goat anti-mouse Ig Ab (Southern Biotechnology Associates Inc., Birmingham, Alabama, USA).

Statistical analysis. The statistical significance of differential findings between experimental groups and control groups was determined by Student's $t$ test. Findings were regarded as significant if two-tailed $P$ values were less than 0.05 .

\section{Acknowledgments}

We thank Gaston Picchio, C. Dolman, and D. Markowitz for excellent technical assistance and Kathy Cairns for editorial assistance with manuscript preparation. H. Zhou is currently a fellow of The Susan G. Komen Breast Cancer Foundation. This study was supported by NIH grant CA83856 (to R.A. Reisfeld), Department of Defense grants DAMD17-02-10562 and DAMD17-02-1-0137 (to R. Xiang), and E. Merck, DarmstadtLexigen Research Center (Billerica, Massachusetts, USA) grant SFP1330 (to R.A. Reisfeld). Scripps Research Institute manuscript number: 16339-IMM.

Received for publication January 20, 2004, and accepted in revised form March 23, 2004.

Address correspondence to: Ralph A. Reisfeld, The Scripps Research Institute, R218, IMM13, 10550 North Torrey Pines Road, La Jolla, California 92037, USA. Phone: (858) 784-8110; Fax: (858) 784-2708; E-mail: reisfeld@scripps.edu.
1. Townsend, A., and Bodmer, H. 1989. Antigen recognition by class I-restricted T lymphocytes. Annu. Rev. Immunol. 7:601-624.

2. Germain, R.N. 1994. MHC-dependent antigen processing and peptide presentation: providing ligands for T lymphocyte activation. Cell. 76:287-299.

3. Darji, A., et al. 1997. Oral somatic transgene vaccination using attenuated S. typhimurium. Cell. 91:765-775.

4. Xiang, R, et al. 2000. An autologous oral DNA vaccine protects against murine melanoma. Proc. Natl. Acad. Sci. U. S. A. 97:5492-5497.

5. Valmori, D., et al. 2000. Induction of potent antitumor CTL responses by recombinant vaccinia encoding and Melan-A peptide analogue. J. Immunol. 164:1125-1131.

6. Shively, J.E., and Beatty, J.D. 1985. CEA-related antigens: molecular biological and clinical significance. Crit. Rev. Oncol. Hematol. 2:355-399.

7. Thompson, J.A., Grunert, F., and Zimmerman, W. 1991. Carcinoembryonic antigen gene family: molecular biology and clinical perspectives. J. Clin. Lab. Anal. 5:344-366.

8. Marshall, J. 2003. Carcinoembryonic antigen-based vaccines. Semin. Oncol. 30:30-36.

9. Tsang, K.Y., et al. 1995. Generation of human cytotoxic $T$ cells specific for human carcinoembryonic antigen epitopes from patients immunized with recombinant vaccinia-CEA vaccines. J. Natl. Cancer Inst. 87:982-990.

10. Xiang, R., et al. 2001. Protective immunity against human carcinoembryonic antigen (CEA) induced by an oral DNA vaccine in CEA-transgenic mice. Clin. Cancer Res. 7(Suppl. 3):856s-864s.

11. Conry, R.M., et al. 1998. Polynucleotide immunization of nonhuman primates against carcinoembryonic antigen. Clin. Cancer Res. 4:2903-2912.

12. Huarte, E., et al. 2002. Enhancing immunogenicity of a CTL epitope from carcinoembryonic antigen by selective amino acid replacements. Clin. Cancer Res. 8:2336-2344.

13. Conry, R.M., et al. 2002. Safety and immunogenicity of a DNA vaccine encoding carcinoembryonic antigen and hepatitis B surface antigen in colorectal carcinoma patients. Clin. Cancer Res. 8:2782-2787.

14. Horig, H., et al. 2000. Phase I clinical trial of a recombinant canarypoxvirus (ALVAC) vaccine expressing human carcinoembryonic antigen and the B7.1 costimulatory molecule. Cancer Immunol. Immunother. 49:504-514.

15. Kawashima, I., et al. 1998. The multi-epitope approach for immunotherapy for cancer: identification of several CTL epitopes from various tumor-associated antigens expressed on solid epithelial tumors. Hum. Immunol. 59:1-14.

16. Keogh, E., et al. 2001. Identification of new epitopes from four different tumor-associated antigens: recognition of naturally processed epitopes correlates with HLA-A*0201-binding affinity. J. Immunol. 167:787-796.

17. Zaremba, S., et al. 1997. Identification of an enhancer agonist cytotoxic $\mathrm{T}$ lymphocyte peptide from human carcinoembryonic antigen. Cancer Res. 57:4570-4577.

18. Clarke, P., Mann, J., Simpson, J.F., Rickard-Dickson, K., Primus, F.J. 1998. Mice transgenic for human carcinoembryonic antigen as a model for immunotherapy. Cancer Res. 58:1469-1477.

19. Eades-Perner, A.M., et al. 1994. Mice transgenic for the human carcinoembryonic antigen gene maintain its spatiotemporal expression pattern. Cancer Res. 54:4169-4176.

20. Thompson, J.A., Eades-Perner, A.M., Ditter, M., Muller, W.J., and Zimmermann, W. 1997. Expression of transgenic carcinoembryonic antigen (CEA) in tumorprone mice: an animal model for CEA-directed tumor immunotherapy. Int. J. Cancer. 72:197-202.

21. Luo, Y., et al. 2003. Plasmid DNA encoding human carcinoembryonic antigen (CEA) adsorbed onto cationic microparticles induces protective immunity against colon cancer in CEA-transgenic mice. Vaccine. 21:1938-1947.

22. Grosenbach, D.W., Barrientos, J.C., Schlom, J., and
Hodge, J.W. 2001. Synergy of vaccine strategies to amplify antigen-specific immune responses and antitumor effects. Cancer Res. 61:4497-4505.

23. Vitiello, A., Marchesini, D., Furze, J., Sherman, L.A., and Chesnut, R.W. 1991. Analysis of the HLA-restricted influenza-specific cytotoxic $\mathrm{T}$ lymphocyte response in transgenic mice carrying a chimeric human-mouse class I major histocompatibility complex. J. Exp. Med. 173:1007-1015.

24. Tangri, S., et al. 2001. Structural features of peptide analogs of human histocompatibility leukocyte antigen class I epitopes that are more potent and immunogenic than wild-type peptide. J. Exp. Med. 194:833-846.

25. Bernhard, E.J., Le, A.X., Barbosa, J.A., Lacy, E., and Engelhard, V.H. 1988. Cytotoxic T lymphocytes from HLA-A2 transgenic mice specific for HLA-A2 expressed on human cells. J Exp. Med. 168:1157-1162.

26. Theobald, M., Biggs, J., Dittmer, D., Levine, A.J., and Sherman, L.A. 1995. Targeting p53 as a general tumor antigen. Proc. Natl. Acad. Sci. U. S. A. 92:11993-11997.

27. Fawell, S., et al. 1994. Tat-mediated delivery of heterologous proteins into cells. Proc. Natl. Acad. Sci. U. S. A. 91:664-668.

28. Kim, D.T., et al. 1997. Introduction of soluble proteins into the MHC class I pathway by conjugation to an HIV tat peptide. J. Immunol. 157:1666-1668.

29. Lu, J., Wettstein, P.J., Higashimoto, Y., Appella, E., and Celis, E. 2001. TAP-independent presentation of CTL epitopes by Trojan antigens. J. Immunol. 166:7063-7071.

30. BenMohamed, L., et al. 2000. Induction of CTL response by a minimal epitope vaccine in HLA A*0201/DR1 transgenic mice: dependence on HLA class II restricted $\mathrm{T}(\mathrm{H})$ response. Hum. Immunol. 61:764-779.

31. Xiang, R., Lode, H.N., Dreier, T., Gillies, S.D., and Reisfeld, R.A. 1998. Induction of persistent tumor-protective immunity in mice cured of established colon carcinoma metastases. Cancer Res. 58:3918-3925. 